\title{
Formes vagabondes. Précarité du médium et pratiques filmiques vernaculaires en cinéma romani
}

Wandering Forms. Medium precarity and vernacular film practices in Romani cinema

\section{Jonathan Larcher}

\section{OpenEdition \\ Journals}

Édition électronique

URL : https://journals.openedition.org/imagesrevues/11350

DOI : 10.4000/imagesrevues. 11350

ISSN : $1778-3801$

Éditeur :

Centre d'Histoire et Théorie des Arts, Groupe d'Anthropologie Historique de l'Occident Médiéval,

Laboratoire d'Anthropologie Sociale, UMR 8210 Anthropologie et Histoire des Mondes Antiques

Référence électronique

Jonathan Larcher, « Formes vagabondes. Précarité du médium et pratiques filmiques vernaculaires en cinéma romani », Images Re-vues [En ligne], 18 | 2021, mis en ligne le 05 octobre 2021, consulté le 16 novembre 2021. URL : http://journals.openedition.org/imagesrevues/11350 ; DOI : https://doi.org/ 10.4000/imagesrevues.11350

Ce document a été généré automatiquement le 16 novembre 2021.

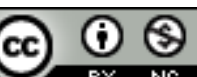

Images Re-vues est mise à disposition selon les termes de la Licence Creative Commons Attribution Pas d'Utilisation Commerciale 4.0 International. 


\section{Formes vagabondes. Précarité du médium et pratiques filmiques vernaculaires en cinéma romani}

Wandering Forms. Medium precarity and vernacular film practices in Romani cinema

Jonathan Larcher

1 Ce texte propose d'interroger plusieurs pratiques filmiques contemporaines qui documentent l'expérience et l'histoire de la mobilité des familles romanis (Roms, Manouches, Gitanos, Gypsies, Travellers, Sinti, Zingari) en Europe occidentale, en faisant de la précarité du médium filmique (ou vidéographique) un ressort plastique et politique. En utilisant, par choix ou par nécessité, des technologies de prises de vue de basse résolution (pour

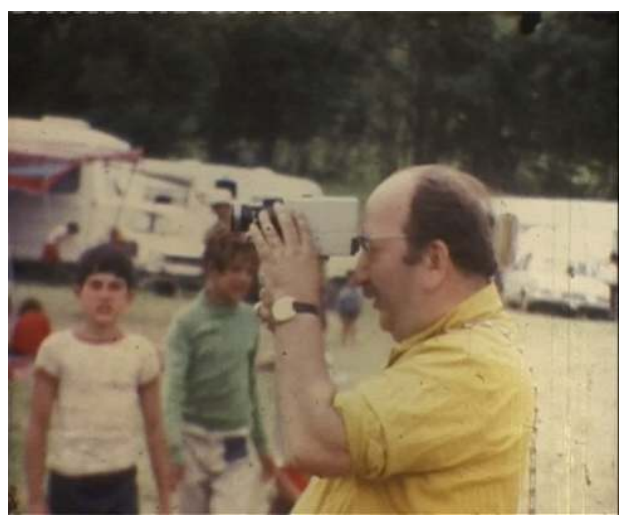
les images argentiques et numériques), ou des supports d'enregistrements faillibles ou périmés, plusieurs cinéastes et artistes ont engagé une réflexion sur les affinités ou les contrastes entre une représentation filmique de la précarité et son médium.

Depuis la fin de la Seconde Guerre mondiale, des cinéastes ont documenté l'expérience du déplacement (migration, itinérance) des familles romanis. Qu'ils soient issus des familles romanis ou de la "société majoritaire $»^{1}$, plusieurs d'entre eux ont développé des pratiques filmiques et des ruses techniques pour élaborer une histoire visuelle critique $^{2}$ qui confronte les positions confortables des cinéastes "gadjé » (non-romanis) et leur esthétisation de la misère des populations roms et tsiganes. Ce texte présente l'analyse de trois pratiques filmiques s'appuyant sur des moyens techniques dits amateurs, des images analogiques de basse résolution ou des "images pauvres" 
numériques, toutes parties prenantes "d'un lumpenprolétariat dans une société de classe des apparences, classées et appréciées [valued] en fonction de leur résolution $»^{3}$. En observant comment les formats réduits, analogiques ou numériques, ont été utilisés, parfois détournés, par les praticiens du cinéma romani, ce travail contribue aux recherches les plus récentes des format studies ${ }^{4}$.

3 Ce texte fait donc l'hypothèse d'une congruence entre formes de vie et formes filmiques ou plastiques - une hypothèse de travail soulevée par l'historien du cinéma Thomas Elsaesser et la théoricienne de la littérature Marielle Macé ${ }^{5}$ - mais aussi celle d'un accord entre ces formes, d'une part, et le format des images, leurs modalités d'inscription sur un support d'enregistrement bien souvent fragile et instable, d'autre part. À la vie d'itinérance, de migration et de circulation des familles romanis répondent ainsi des formes filmiques et des formats vagabonds. En prêtant attention aux gestes des cinéastes, aux technologies employées, ainsi qu'aux situations filmées, ce texte propose d'observer comment certains formats, numériques ou analogiques, de l'image en mouvement, se sont révélés pertinents pour rendre compte d'une expérience de la mobilité et acquérir la confiance des personnes filmées. L'histoire de ces formes vagabondes se fait en gardant à l'esprit la spécificité de chaque format utilisé tout en se « focalis[ant] sur ce qu'il y a en dessous, au-delà ou derrière les objets qui recèlent et véhiculent ${ }^{6}$ les images.

4 La présente analyse appréhende donc le cinéma romani moins par l'identité de ses auteurs que par les technologies vidéo et filmiques employées et par une recherche de formes filmiques rendant compte des circulations et migrations des familles roms et tsiganes. Cette traversée du cinéma romani, distincte d'une littérature traitant essentiellement de cinéastes reconnus ${ }^{7}$, soulève trois enjeux, traités au fil du texte. Ancrées dans une économie domestique et vernaculaire, ces pratiques filmiques emploient des tactiques qui échappent aux chaînes opératoires de l'industrie du cinéma, pour au contraire se développer dans un usage convivial des outils filmiques. Cette utilisation reprend les observations et propositions d'Ivan Illich sur la " recherche conviviale» dans laquelle des individus "travaillent seuls ou en équipes très réduites, visant au premier chef, par leur étude, à façonner directement leur mode de vie $»^{8}$. Les œuvres retenues s'inscrivent également dans un mouvement de l'art contemporain romani qui "récupère " (to reclaim) le campement, le cosmopolitisme et l'avant-garde comme des formes de vie et des dimensions essentielles de l'histoire des groupes romanis ${ }^{9}$. Enfin, l'étude de la genèse des films, ainsi que l'analyse filmique et l'observation fine des copies filmiques (ici des positifs Super 8), argue en faveur de l'adoption d'une approche conciliant archéologie des média et anthropologie. Ce texte souhaite ainsi formuler des hypothèses de travail pour une anthropologie des Roms et Tsiganes, en rapprochant des expériences et des conjonctures relevant très souvent de corpus ethnographiques et historiographiques distincts.

5 Ce parcours, en trois étapes ${ }^{10}$, s'ouvre avec la chronique filmique réalisée par l'écrivain et pasteur tsigane Matéo Maximoff, des années 1960 jusqu'au courant des années 1990. Dans un deuxième temps, le texte décrit les tactiques et pratiques des plasticiens et cinéastes «gadjé » qui, au tournant des années 2000 et 2010, rencontrent des familles romanis dans les interstices des métropoles d'Europe de l'Ouest (en France et en Espagne). Cette déambulation s'achève, enfin, avec les courts métrages produits par les artistes romanis anglais Delaine Le Bas et Damian James Le Bas qui, à la marge de leur œuvre plastique et littéraire, ont constitué un véritable laboratoire vidéographique. 


\section{Les chroniques filmiques de Matéo Maximoff} manouche, Matéo Maximoff est un écrivain, conférencier et pasteur pentecôtiste. Il est l'auteur de plusieurs œuvres de fictions et d'articles publiés dans des revues spécialisées (Études tsiganes, The Journal of the Gypsy Lore Society) qui présentent l'histoire des groupes romanis, des traditions orales aux traumas de la Seconde Guerre mondiale. De sa naissance jusqu'à la fin des années 1930, Matéo Maximoff circule à travers la France (et même l'Espagne où il passe une partie de son enfance). En compagnie de sa famille maternelle, Matéo Maximoff s'initie à la pratique du cinéma forain, au point d'acquérir une parfaite maîtrise des outils cinématographiques ${ }^{11}$.

Emprisonné en $1938^{12}$ à la suite d'une rixe impliquant sa famille et un autre groupe romani, puis interné dans plusieurs camps français jusqu'en 1943, il apprend à lire et à écrire de façon autodidacte. Après la Libération, il s'installe en 1944 avec sa famille en proche banlieue parisienne - entre Montreuil et Noisy-le-Sec. Il fait paraître son premier roman, Les Ursitory, en 1946, et devient ainsi le « premier écrivain tsigane » de renommée internationale. Proche de plusieurs artistes, écrivains et cinéastes (dont ceux qu'il a fréquentés sur des tournages avant la guerre), Matéo Maximoff s'inscrit pleinement dans une "culture de contact » qui se forme entre les mondes sociaux du cinéma et ceux des familles romanis ${ }^{13}$. Ceci lui permet de rapidement devenir un intermédiaire pour tous les photographes, cinéastes ou journalistes voulant approcher les familles roms et tsiganes de la banlieue parisienne. En 1980, Matéo Maximoff, converti au pentecôtisme depuis 1962, donne son témoignage dans l'émission « $\mathrm{Au}$ rendez-vous des Tziganes » de la Radio Tzigane, et revient sur son parcours atypique de cinéaste, en commençant par son rôle de figurant dans le film Gloria (1931) :

À quatorze ans, j'ai commencé à tourner en tant que figurant. Or l'acteur principal de ce film, il s'appelait André Luguet. Le second acteur s'appelait un certain Jean Gabin (sic.). [...] Et je suis ainsi devenu cinéaste, d'abord petit figurant, puis des petits rôles, puis ensuite technicien. Et pendant 31 ans, tous les films qui ont été tournés en France sur les Tsiganes l'ont été avec ma participation. J'ai tourné avec les plus grands metteurs en scène français : Jean Renoir, Christian Jacque, et tant d'autres. Mais tous ces talents que j'avais pour le monde, je les mets maintenant au service de Dieu. C'est ainsi que depuis 8 ans - oh, en tant qu'amateur, puisque j'ai une caméra Super 8 - j'ai tourné 85 films à travers les conventions et à travers 31 pays, où je suis allé soit seul, soit avec quelques membres de la Mission Tzigane ${ }^{14}$.

Par la mise en récit de son expérience au sein de l'industrie du cinéma, Matéo Maximoff rend compte de sa déambulation dans des mondes sociaux du cinéma extrêmement contrastés, sans pour autant déconsidérer son statut de "cinéaste amateur " L'accumulation de ces expériences et de ce savoir du cinéma trouve en effet son accomplissement dans ces " 85 films». Bien sûr, ce témoignage montre également comment son engagement au sein du mouvement pentecôtiste s'est traduit par une reconversion de savoirs et d'aptitudes, mis au service de sa communauté d'appartenance (à la fois d'origine - les groupes romanis - et d'élection - la Mission évangélique des Tziganes de France ${ }^{16}$. La pratique filmique de Matéo Maximoff est en effet indissociable de ses engagements religieux et politiques. Il rejoint le mouvement pentecôtiste en 1962, au moment même où son essor a pris une dimension internationale avec des missions d'évangélisation et des conventions organisées dans 
toute l'Europe (occidentale), tout en s'engageant, dans la Communauté Mondiale Gitane, une organisation politique qui deviendra au fil des années le Comité International Tzigane puis en 1971, le Comité International Rom. Qu'ils soient au service de la mission ou des collectifs politiques, sa pratique et ses films développent toutefois un style éminemment personnel, fait de bricolages et de mise en scène de soi devant l'objectif de sa caméra. Des compétences techniques et actorales acquises au fil de sa pratique du cinéma forain et lors des tournages de fiction.

Fig. 1

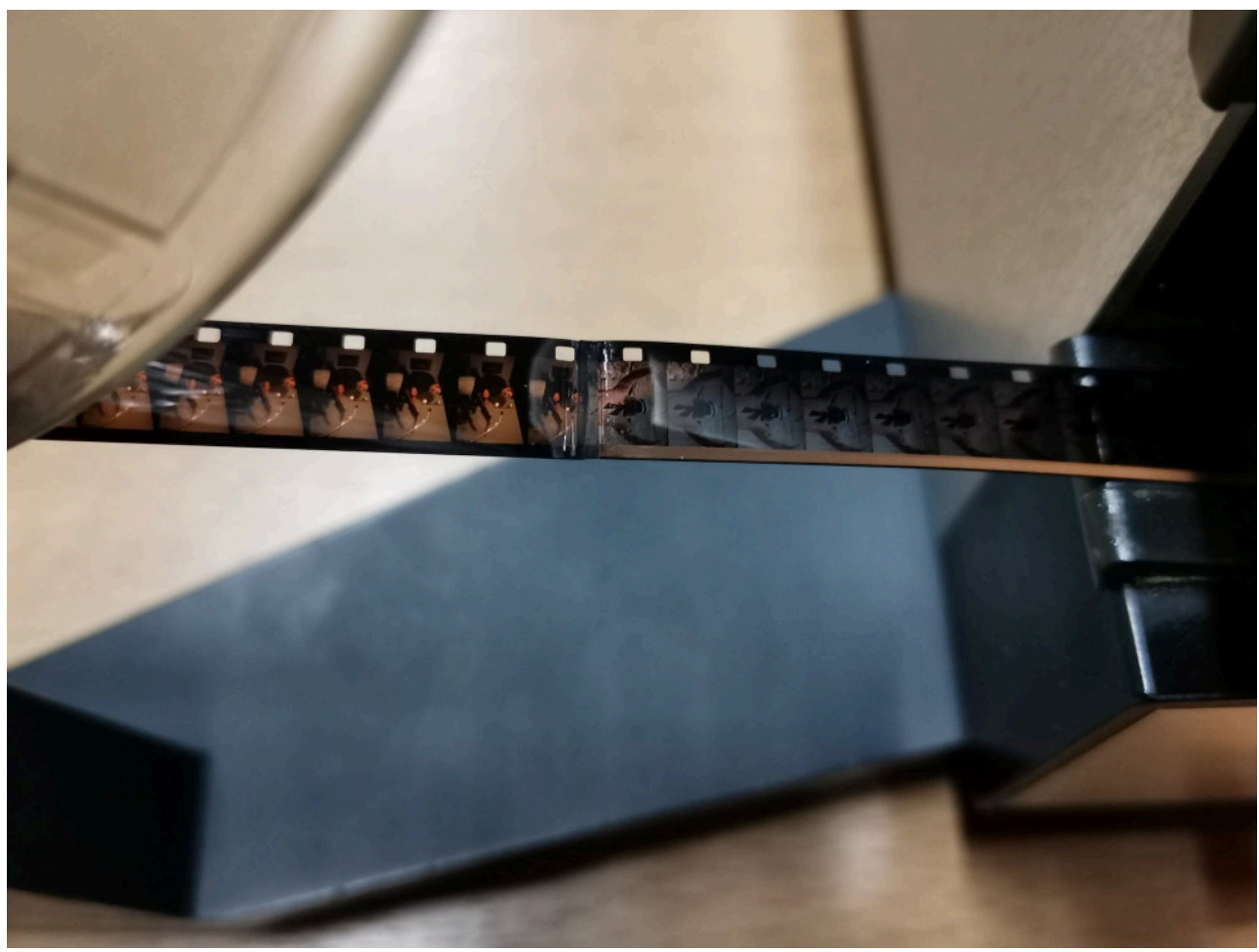

Photographie du film Radio Tzigane de Matéo Maximoff (date inconnue). Cliché pris à la Médiathèque Matéo Maximoff-Fnasat-Gens du Voyage.

Jonathan Larcher

$\mathrm{Au}$ cours de ses nombreux voyages et déplacements, Matéo Maximoff réalise une importante documentation visuelle, compilant des rushes tournés en Super 8 Kodachrome ${ }^{17}$ lors des grandes conventions pentecôtistes, des voyages avec la Mission tzigane (à des fins de missionnaires ou de "retraite spirituelle»), à l'occasion de manifestations politiques, ou au quotidien avec ses proches de l'Est parisien. Une fois les images développées en laboratoire, Matéo Maximoff effectue le montage avec grand soin dans son petit pavillon de Noisy-le-Sec. Plusieurs indices montrent qu'il a constamment travaillé sur ses films: révisant les montages, compilant les rushes et inventant les titres au fil des projections. Sur les 85 films mentionnés dans l'émission radio de 1980, le fonds de la Médiathèque Matéo Maximoff-Fnasat-Gens du Voyage ne compte que 73 bobines. Chacun des « films montés » tenant sur une même bobine de 80 à 160 mètres [durant entre 15 et 30 minutes], un premier examen des archives montre que certains films semblent incomplets ou répartis sur plusieurs bobines, et surtout que les titres font souvent défaut au début des films (ou sur les boîtes). Ainsi, parmi les films importants mentionnés par Matéo Maximoff dans son journal, énumérant les 
titres repris par ses biographes et proches, on ne trouve nulle trace des films Avec l'aide de Dieu, achevé en 1975, et Sourires de l'Inde, aujourd'hui conservé au sein du fonds audiovisuel déposé à la Médiathèque. Il est possible que Matéo Maximoff ait fait un premier tri dans ses films (comme il l'a fait pour les 19375 diapositives numérotées dont on ne recense aujourd'hui que $8000^{18}$ ). Une observation plus fine des films sur la visionneuse utilisée par Matéo Maximoff lui-même révèle sa capacité à assembler des éléments hétéroclites au gré des montages (Fig.1) associant, par exemple, un film Super 8 sonore à droite, et un film muet du même format à gauche.

Fig. 2

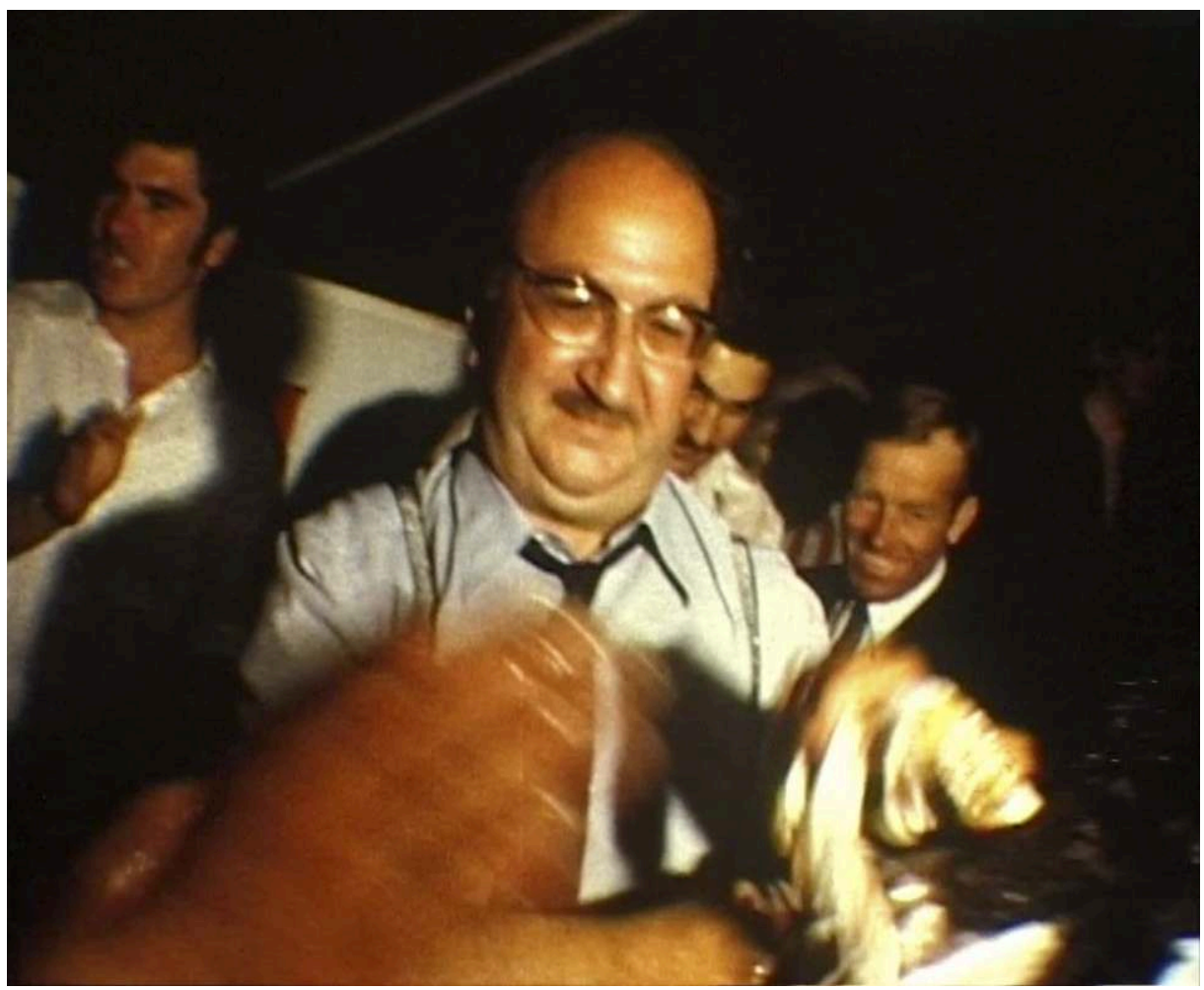

Matéo Maximoff, Les Roms, date et titre incertains. Maximoff s'apprête à faire baptiser un fidèle. 
Fig. 3

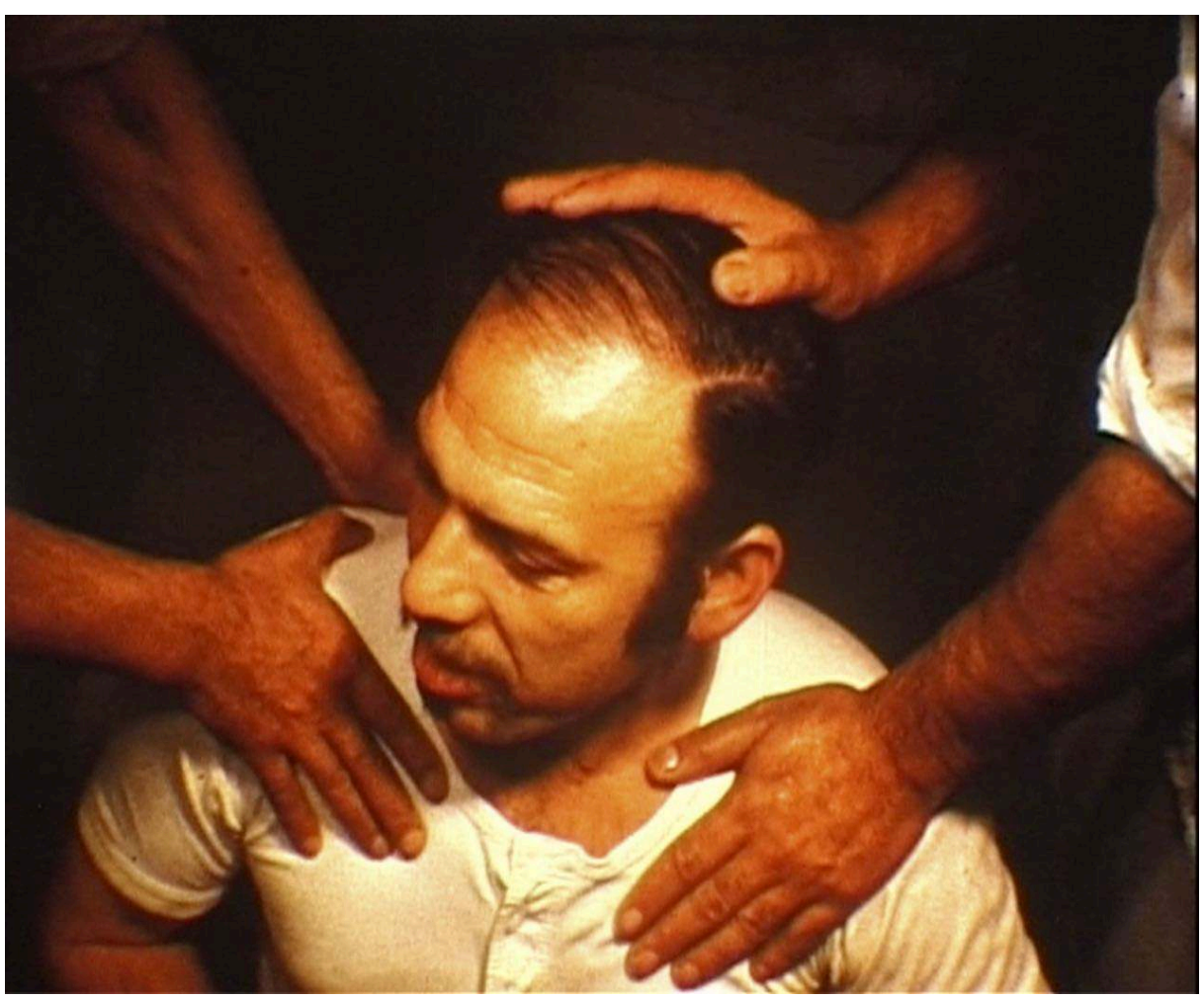

Matéo Maximoff, Les Roms, date et titre incertains. C'est probablement lui qui est derrière la caméra.

10 Ce qui ici est techniquement inhabituel, dans la mesure où la vitesse de tournage et de projection des deux films est différente: 18 images/seconde pour le film muet, 24 images/seconde pour le film sonore. Cette liberté prise avec le médium correspond, dans les situations filmées, à son extraordinaire aptitude à se tenir alternativement derrière et devant la caméra. En effet, l'une des marques de ses films - comme de ses photos - est précisément cette faculté à se mouvoir et à reproduire les gestes des rituels et des interactions aussi bien dans le champ de l'image (prêchant, baptisant, priant, tenant la pose avec les pasteurs et les camarades de lutte) que derrière la caméra. Maximoff se tient par ailleurs très souvent au plus près des familles, des fidèles des pasteurs ou des responsables politiques ${ }^{19}$. Il peut être ainsi filmé assistant une jeune fille qu'il vient de baptiser par immersion avant de repasser derrière la caméra pour filmer la suite du rituel (Fig. 2 et 3).

11 Si cet extraordinaire corpus documentaire a pu être élaboré d'abord à des fins d'édification, de formation, et même d'évangélisation, suivant les velléités du mouvement ${ }^{20}$, il n'en est pas pour autant réductible à une production de l'association Vie et Lumière ${ }^{21}$. Les films réalisés lors des conventions se révèlent être moins des films confessionnels, qui s'inscriraient dans des «dispositifs de médiatisation intégrés à l'action cérémonielle ${ }^{22}$, que de fines descriptions filmiques de l'ensemble des interactions et des travaux réalisés en marge de la liturgie des cultes. La légitimité de Matéo Maximoff, et le respect dont il bénéficie parmi les groupes romanis lui permettent de filmer les situations et les interactions sociales au plus près - comme il le fait d'ailleurs pour les siens, la kumpania des Roms Kalderash de l'Est Parisien ${ }^{23}$. En s'écartant à de nombreuses reprises de la scénographie des rituels politiques et religieux, il n'éprouve aucune difficulté à faire entrer dans le champ de l'image les 
conditions matérielles d'existence des communautés romanis en Europe, documentant aussi les conditions matérielles d'existence précaires et la paupérisation de nombreux groupes roms et tsiganes en Europe. Comme l'attestent plusieurs légendes des diapositives, rédigées par ses soins entre 1984 et 1989 dans un catalogue, un tapuscrit de 170 pages, ces signes de précarité n'échappent pas à son regard aguerri :

173 - les enfants sont toujours beaux

174 - Et souriants

175 - Mais certains sont nus, vu la chaleur

176 - Cette tente est misérable. Et dire que je suis né peut-être dans une tente comme celle-ci ? $^{24}$

Fig. 4

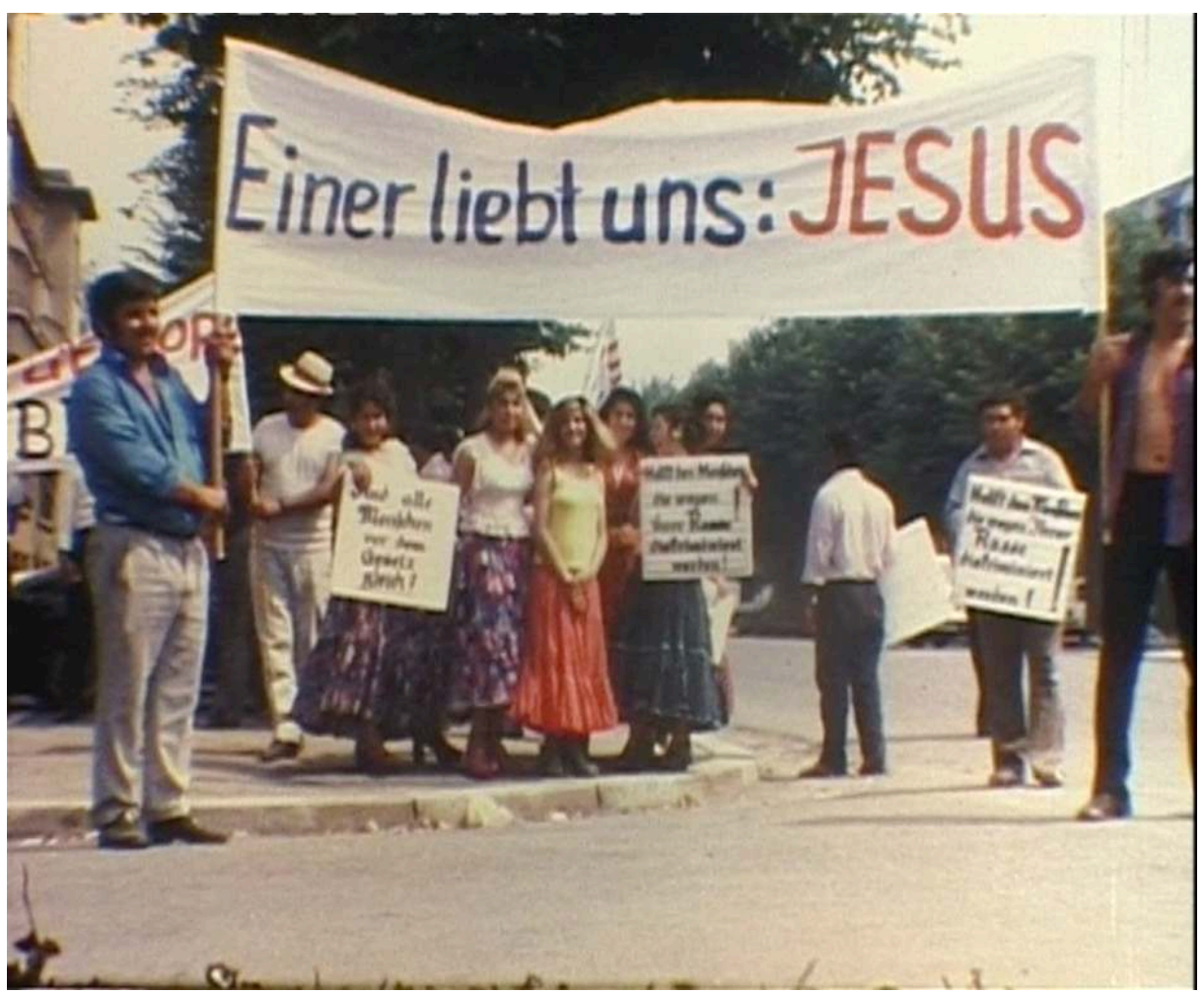

Matéo Maximoff, Quelques manouches, date incertaine. Manifestation en marge d'une convention en Alsace ou en Allemagne. 
Fig. 5

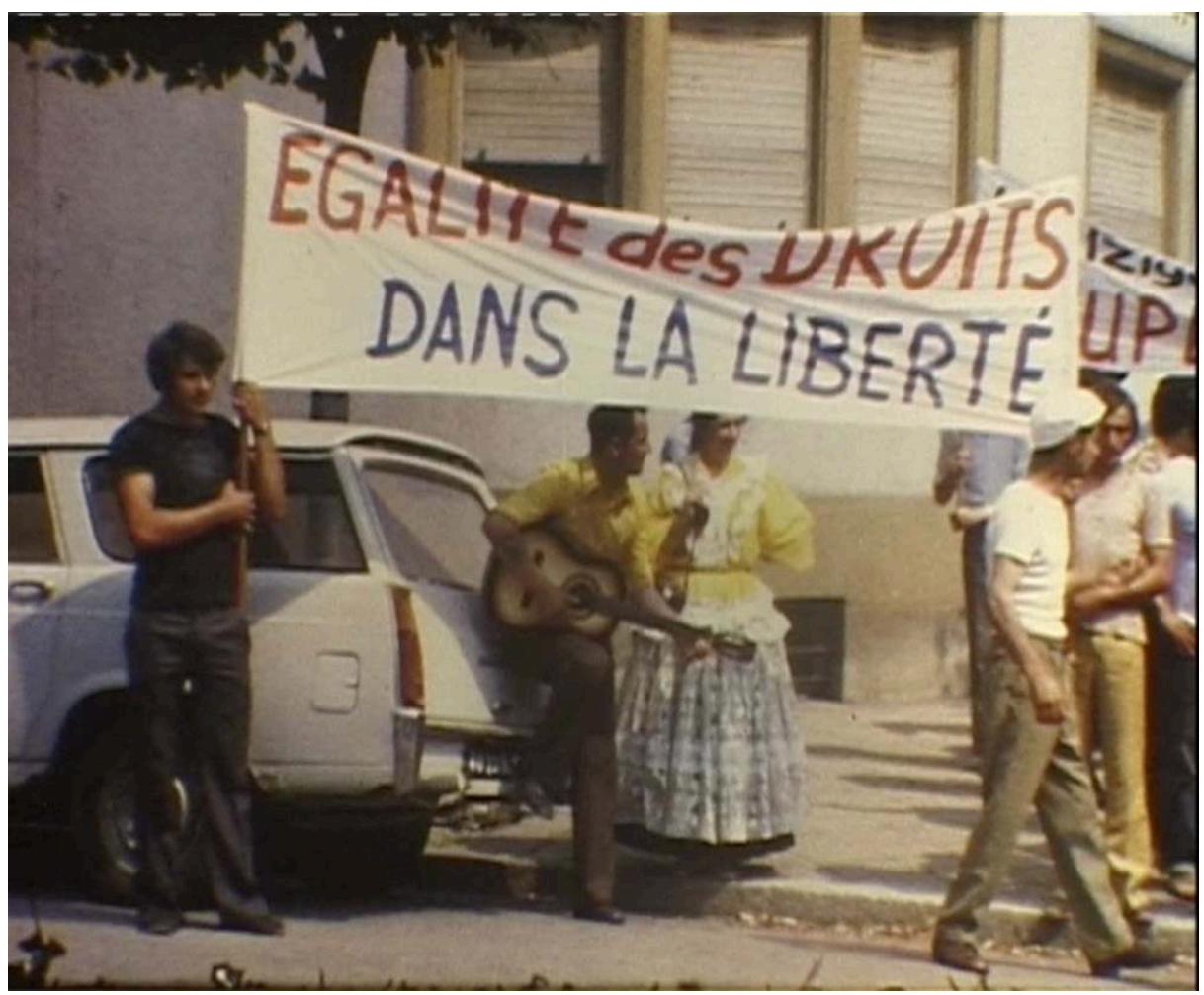

Matéo Maximoff, Quelques manouches, date incertaine. Manifestation en marge d'une convention en Alsace ou en Allemagne.

Pour autant, ces mentions sont rares. Les notes de son journal et les légendes s'attardent plutôt sur une description du contexte et sur une déclinaison précise de l'identité de ses interlocuteurs: leur nom - et surtout leur surnom, c'est-à-dire la manière dont ils sont connus par les Roms - ainsi que des bribes de leur généalogie ou de leurs alliances. Les films de Matéo Maximoff constituent ainsi une formidable chronique audiovisuelle de la circulation des hommes et des femmes romanis dans le contexte de l'émergence du mouvement pentecôtiste tsigane et d'un mouvement politique international rom. La frontière entre les deux n'était d'ailleurs pas aussi hermétique qu'aujourd'hui. Une manifestation organisée par la mission pouvait ainsi exiger la reconnaissance du génocide des familles tsiganes par l'Allemagne nazie et leur internement par le régime de Vichy tout en faisant cohabiter sur leurs pancartes des slogans comme : «Quelqu'un nous aime : Jésus » et "égalité des droits dans la liberté » (Fig. 4 et 5). Cette histoire intriquée de la construction confessionnelle d'une présence des Tsiganes et Roms dans l'espace public (entre 1950 et 1990) a cédé la place à une reconfiguration des collectifs politiques roms après la chute du mur de Berlin et la résurgence d'un "problème Rom » à l'échelle européenne, dans un contexte marqué par la circulation des Roms des Balkans et l'expansion de l'Union européenne.

\section{Migration et tactiques du film argentique chez Ben Vine, Gee-Jung Ju et Pilar Arcila}

13 Dès le milieu des années 1990, la migration des Roms roumains en Europe occidentale devient un thème important des représentations médiatiques et des politiques 
publiques plus particulièrement tournées vers la question de l'habitat précaire. La réalisation des films documentaires, de plus en plus nombreux à se confronter au "Problème rom ", suit le plus souvent cette temporalité de l'urgence politique. Parmi cette somme de productions audiovisuelles, une poignée de films et de reportages élaborés en France et en Espagne se distinguent par un usage du film Super 8. Ben Vine, Gee-Jung Ju et Pilar Arcila, trois photographes et cinéastes qui ont abondamment travaillé avec le médium argentique, à des fins éthiques et tactiques ${ }^{25}$, ont documenté l'expérience vécue de la migration pendulaire des Roms roumains et ses corollaires travail de rue, bidonvilles, économie de survivance - et engagé leurs pratiques filmiques dans différentes directions critiques. Confronté à la précarité des groupes romanis, chaque cinéaste s'est employé à trouver une position tout en recherchant et inventant des formes conviviales, avec une économie de moyen et de financement ${ }^{26}$.

Fig. 6

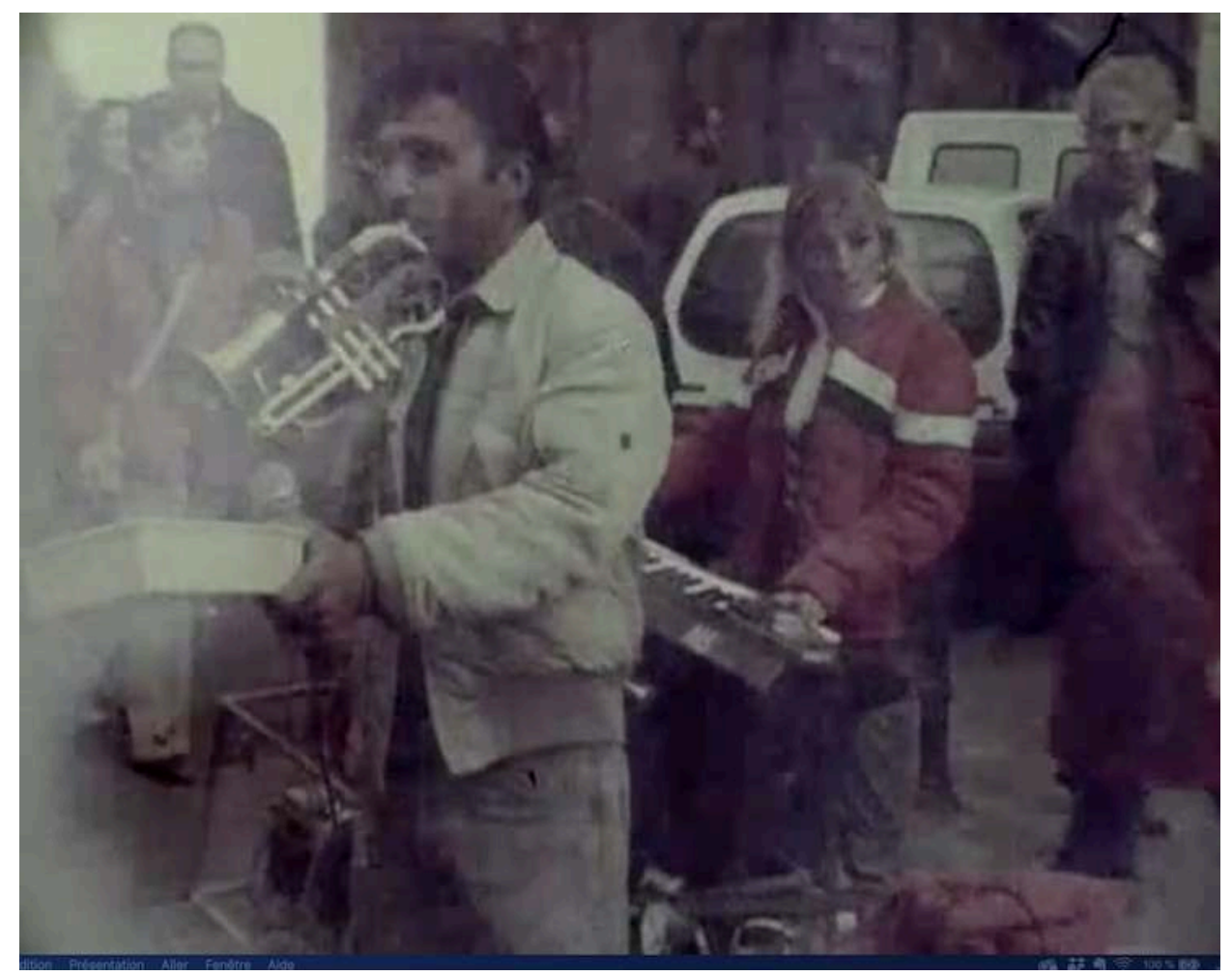

Ben Vine, Olvido, 2008.

Le premier geste est celui du photographe et artiste visuel Ben Vine qui, au milieu des années 2000 , réalise un court métrage constitué d'un seul plan séquence centré sur des musiciens de rue rencontrés dans son quartier, à Barcelone. Tourné avec une bobine périmée de film Super 8 muet (couleur), achetée sur le même marché où performe le couple de musiciens, Olvido (2006) fait concorder la fragilité du médium filmique avec la précarité des conditions d'existence des familles romanis circulant entre l'Europe de l'Ouest et la Roumanie (Fig. 6). Dans les quelques lignes qui accompagnent le film lors de ses programmations (ou sur son compte Vimeo), Ben Vine explique ainsi cette démarche : "Olvido est une réflexion sur le statut ambigu et le rôle de ces musiciens de rue dans la société espagnole. En interrogeant également mon propre regard, ce film est une réflexion sur ce que la société considère comme art, comme culture et comme 
rebut. Des notions qui s'entrelacent $»^{27}$. Son travail sur la « défectibilité $»^{28}$ du Super 8 se situe dans le prolongement des travaux du cinéma expérimental, qui subit depuis les années 1990 la rapide obsolescence de ses outils de productions ${ }^{29}$, tout en interrogeant la manière dont cette obsolescence contribue ou fait obstacle à la reconnaissance d'une œuvre d'art. S'il reconnaît que l'usage de la pellicule favorise la reconnaissance de son travail, il dresse aussi le constat lucide qu'elle ne semble pas opérer de la même manière pour les sujets filmés.

Dans un bidonville de Lyon, Gee-Jung Jun réalise son premier court métrage (France $2007,2007)$ sur support argentique en utilisant un film $16 \mathrm{~mm}$ noir et blanc (et muet). Développé de manière artisanale, le grain de la pellicule est rendu apparent. À l'opposé d'une stratégie de retrait, qui consiste à se tenir en observateur à la marge des situations sociales, le cinéaste filme et monte une série de plans formellement identiques où la relation entre les personnes filmées et le « filmant » est saillante voire même centrale ${ }^{30}$. Les uns après les autres, hommes, femmes, enfants et vieillards se tiennent sur le seuil de leur baraquement (leur baracă), face à l'objectif, pendant plusieurs dizaines de secondes, immobiles ou se jouant des mauvais tours devant la caméra. Dans cette frontalité et cette scénographie minimale des situations, les familles parviennent à progressivement symétriser la relation filmante, s'adressant avec malice au cinéaste, s'appropriant le dispositif « sérieux » du portrait photographique, pour le soumettre à leurs représentations.

16 Au cours de cette même période, dans les années 2000, la ville de Marseille est le théâtre de déplacements continus des bidonvilles de Roms roumains, opérés en parallèle de la mise en place d'un vaste projet de «rénovation» urbaine. Engagé une décennie plus tôt, le projet Euroméditerranée se voit renforcé par l'élection de Marseille en 2009 au titre de "Capitale Européenne de la Culture » pour l'année $2013^{31}$. C'est au sein de ce double arc historique que naît le projet du film de Pilar Arcila (Le Pendule de Costel, 2013). Sa rencontre avec Costel, un Rom du Nord-Ouest de la Roumanie, prend tout d'abord une direction similaire au film Olvido de Ben Vine :

Au printemps 2006, je venais d'emménager dans le centre-ville de Marseille lorsque j'ai commencé à voir des hommes et des femmes arpenter les rues avec des poussettes sans bébés et chargées de bricoles. L'image de gens cherchant dans les poubelles des choses à recycler m'était familière car en Colombie, où je suis née, le besoin a poussé un certain nombre de personnes à vivre du recyclage des déchets depuis très longtemps. Le décalage entre la dureté de la tâche et l'imaginaire évoqué par la poussette m'interpellait sans cesse. [...] Après plusieurs visites au squat en compagnie de Cécile qui traduisait nos échanges, j'ai proposé à Costel de le filmer lors de ses déambulations dans la ville, j'avais l'idée d'un film court, une ballade en Super 8 N\&B sur fond de rénovation urbaine. Je voulais montrer le contraste entre l'opération de « rénovation » du centre de Marseille qui poussait les habitants pauvres à la périphérie et l'installation dans les interstices du centre-ville d'autres exclus de la société qui dévoilaient la misère qu'on voulait cacher ${ }^{32}$.

17 Nouant des liens de confiance avec Costel et sa famille, Pilar Arcila va pourtant orienter son projet dans une autre direction. La réalisation de ces images Super 8 et le prêt d'une caméra numérique semi-professionnelle lui permettent en effet d'établir une relation avec les sujets filmés qui tranche avec une écologie des images saturée de productions médiatiques sur les familles roms du quartier :

La proximité [du squat de la famille de Costel] avec La friche «Belle de Mai » [...] (ancienne usine de tabac, squatté par des artistes puis transformée en lieu culturel marseillais) [...] et un studio de télévision avait attiré beaucoup de monde, touché 
par la précarité de leur situation, scandalisé par la proximité de cette misère, attiré par leur côté exotique. Leur présence a commencé à être médiatisée, ils ont été filmés à leur insu depuis les studios de Plus Belle la Vie, leurs voisins du milieu de la culture sont venus et très vite ils sont devenus des objets d'attirance et de refus. Des photographes, des journalistes, des artistes, des membres d'associations, des politiciens sont venus parler pour eux, les prendre en photo... Il y a eu comme une sorte de concurrence sur la légitimité d'une présence à leur côté, agrémenté des cadeaux pour obtenir leur grâce. J'avais l'impression d'une réunion de vautours se partageant le spectacle de la misère ${ }^{33}$.

Ces motifs repoussants et attirants du paria et de la misère exotique des Roms et Tsiganes sont en réalité une survivance (dans les productions visuelles contemporaines) d'un archétype qui émerge avec l'essor du journal imprimé au XIX siècle, avant de se démultiplier par l'intermédiaire du reportage social, des massmédias d'informations et du cinéma documentaire ${ }^{34}$. Cet archétype se double toutefois d'une représentation de la relation filmante comme une préhension. Dans sa rencontre avec la famille de Costel, Pilar Arcila se retrouve en effet confrontée à une situation qui s'apparente à un marché audiovisuel. Le bidonville, occupé principalement par des Roms d'origine roumaine est littéralement envahi par des cinéastes et des artistes, qui veulent faire «leur» film ou « leurs » images autour de ces familles. Dans un contexte d'hyper-visibilité des migrations pendulaires roumaines, les familles roms craignent, voire même critiquent, la possibilité d'une exploitation économique de leur image. La caméra Super 8, avec ses images silencieuses et en noir et blanc, écarte cette éventualité. De cette façon, il est évident pour tous que la recherche d'un gain immédiat ne correspond nullement à sa démarche ${ }^{35}$.

Fig. 7

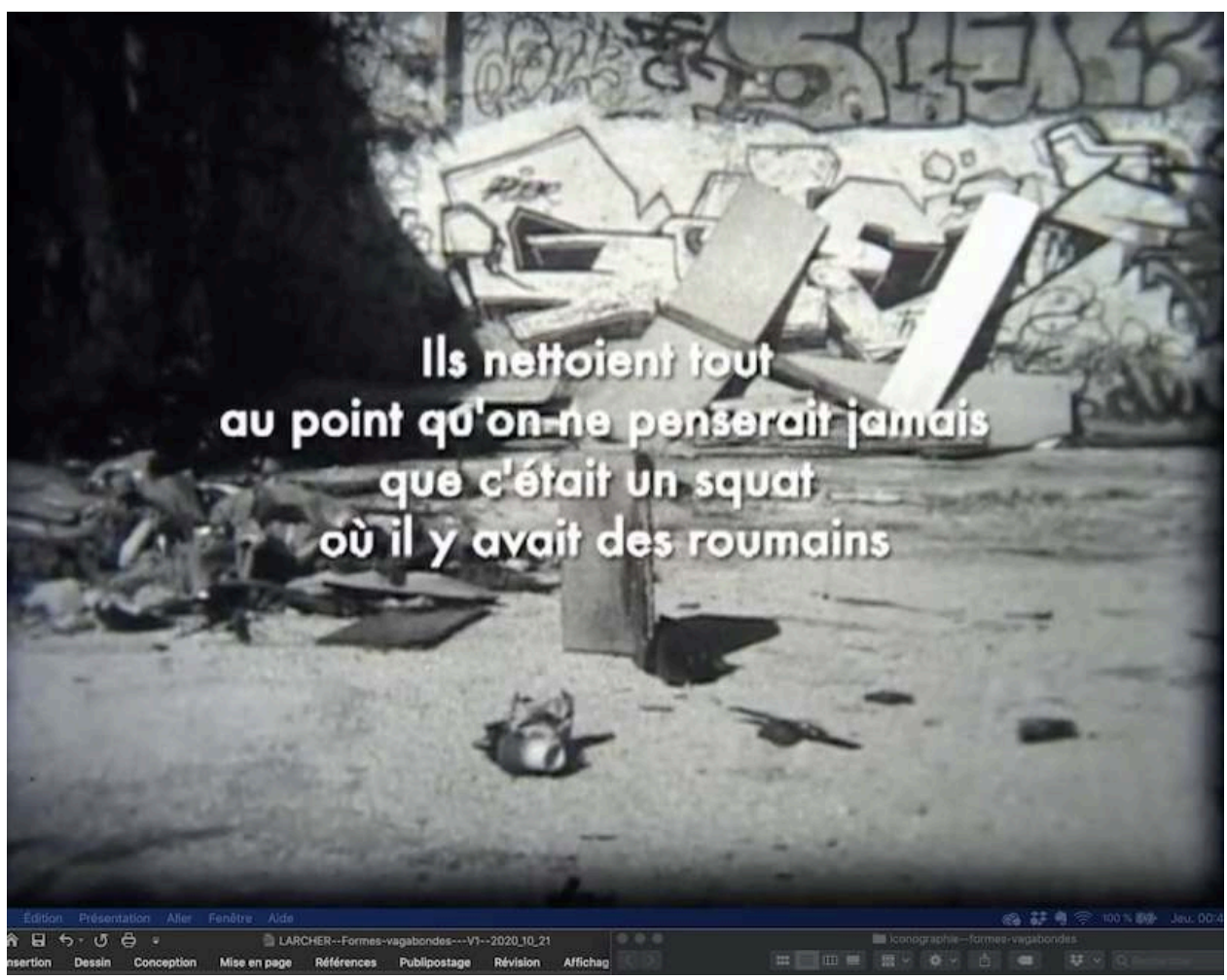

Pilar Arcila, Le Pendule de Costel, 2013. 
Si, selon ses mots, Pilar Arcila utilise les films Super 8, muet, noir et blanc, pour rompre d'abord avec le misérabilisme et "l'impersonnalité des images vidéo ${ }^{36}$, ce versant critique se complète toutefois d'un versant plus réflexif sur les puissances descriptives de l'image de basse résolution. Les premières séquences du film en noir et blanc s'attachent en effet à décrire le travail de Costel, son rythme et ses gestes de chiffonnier, en regard de l'entreprise, violente, de destruction et de reconstruction du centre-ville marseillais. Ce contraste entre les images de Costel déambulant avec sa poussette et celles du démantèlement des bidonvilles et des quartiers historiques par des bulldozers (et un dispositif policier toujours présent) donne aussi naissance à une forme dialogique qui se déploie tout au long du film. Au centre des images noir et blanc, Pilar Arcila reprend sous formes de courtes phrases les paroles et les réflexions de Costel sur la pratique de la chine et la destruction du quartier (Fig. 7).

Fig. 8

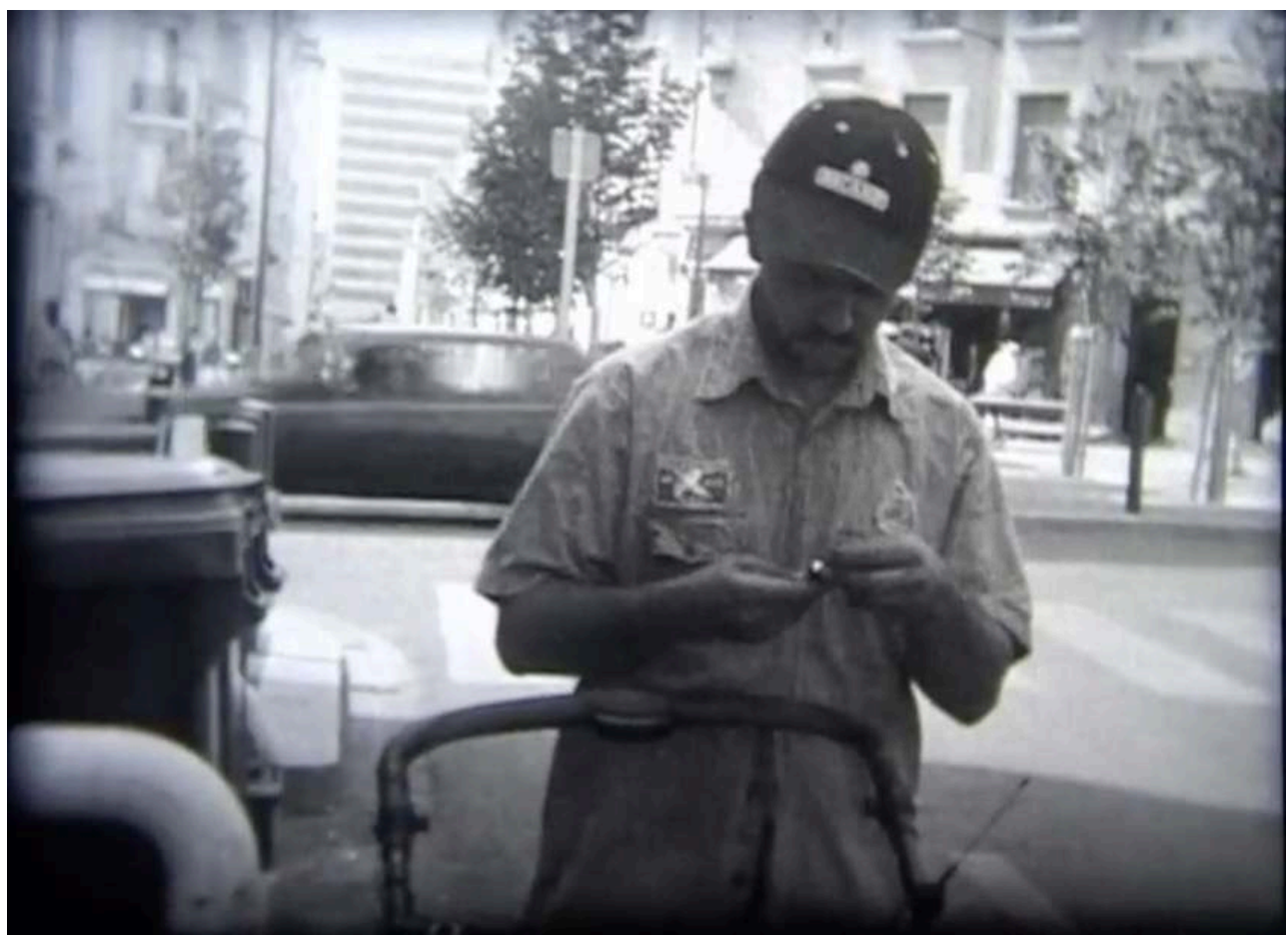

Pilar Arcila, Le Pendule de Costel, 2013. Costel filmé en Super 8 par Pilar Arcila. 
Fig. 9

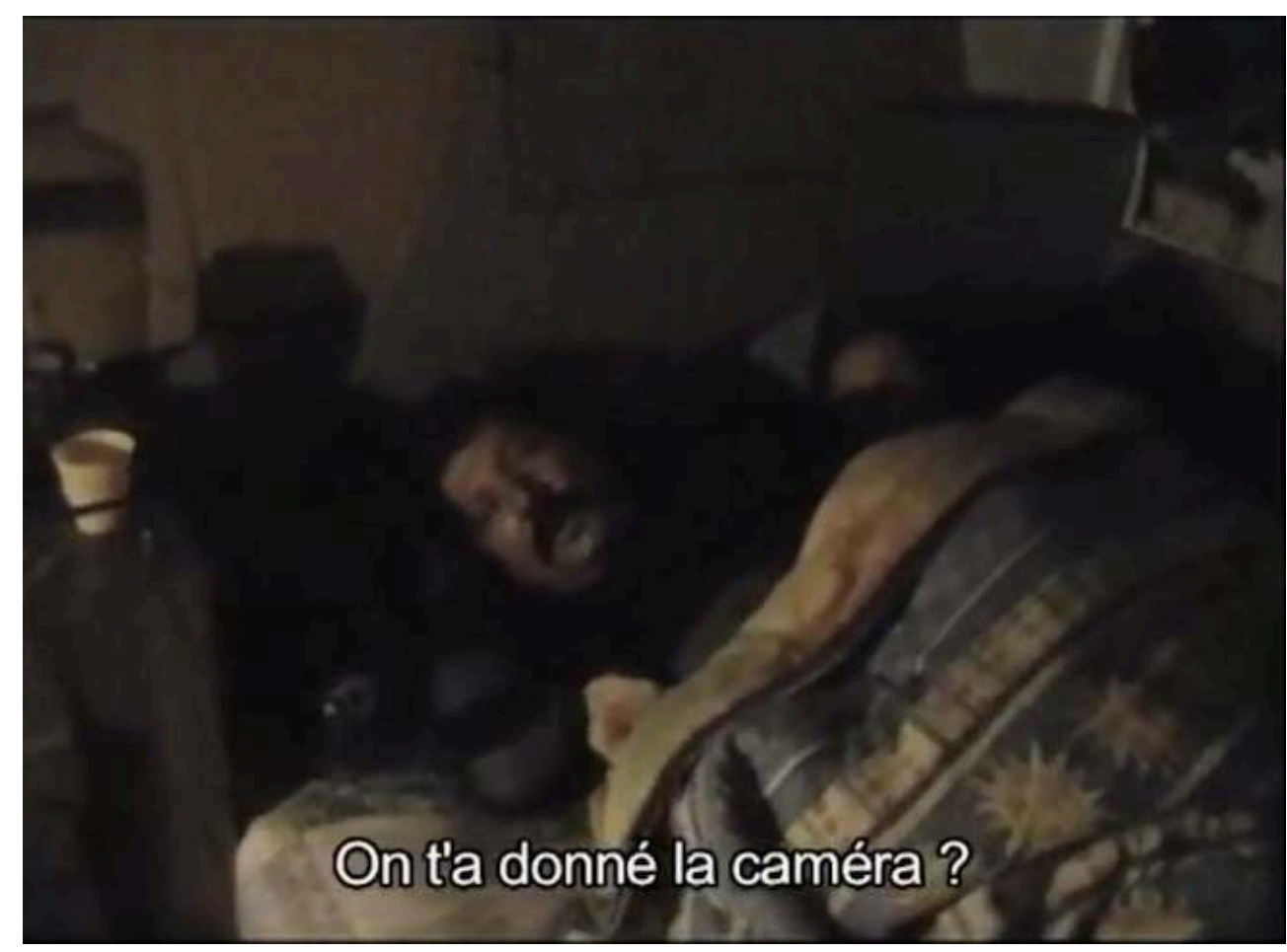

Pilar Arcila, Le Pendule de Costel, 2013. Costel filmant ses voisins avec une petite caméra numérique.

Ce dialogue, qui introduit le film, est en réalité l'aboutissement, en salle de montage, d'une longue et progressive collaboration entre la cinéaste et Costel. C'est en effet à la demande de ce dernier que Pilar Arcila lui confie une caméra numérique semiprofessionnelle pour filmer la vie quotidienne de sa famille - dans l'idée d'établir une correspondance avec les proches restés en Roumanie. En dérushant les images pour en produire des copies pour Costel, Pilar Arcila découvre une séquence vidéo qui sera, pour elle, la genèse de la forme du film, faisant dialoguer les images argentiques de la cinéaste et celles, numériques, de Costel (Fig. 8 et 9). En travaillant avec deux technologies de basse résolution, la cinéaste prend soin d'attribuer une véritable légitimité plastique et descriptive aux images de son interlocuteur. De la sorte, l'amplitude et la profondeur des deux images forment une véritable parité plastique. Un engagement dont Nicole Brenez a très justement identifié la "haute densité tant éthique que plastique $»^{37}$ :

Les initiatives de Jean Rouch et du cinéma direct (en particulier canadien) ont créé une riche tradition de formes collaboratives et participatives avec les sujets filmés. Mais on peut parler désormais de formes conviviales au sens d'Ivan Illich, en découvrant les œuvres de plus en plus nombreuses qui travaillent à mettre en circulation les images d'autrui, à l'instar de Clarisse Hahn (Prisons - notre corps est une arme, 2012, pour des activistes kurdes), Bijan Anquetil (La nuit remue, 2012, pour des réfugiés afghans) ou Pilar Arcila (Le Pendule de Costel, 2013), pour une famille rom $^{38}$. 


\section{Traveling images : le cinéma vernaculaire de Delaine Le Bas \& Damian James Le Bas}

Ces inventions formelles d'un cinéma romani, qui prend pour sujet principal l'expérience de circulation et de migration, naissent aussi dans un contexte marqué par la volonté des artistes, militants et universitaires romanis de coordonner la production, la diffusion, la patrimonialisation et la conservation de leurs œuvres ${ }^{39}$. Ce désir, formulé notamment au sein de la Central Europe University en 2014 et 2015, prend la forme d'un ensemble de propositions pour impliquer davantage les artistes et chercheurs d'origine romani dans les travaux, expositions et politiques publiques les

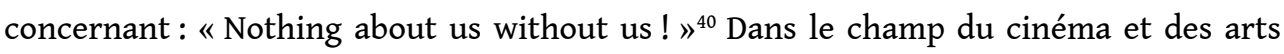
visuels, ce mouvement prend la direction d'une «souveraineté visuelle»: il aboutit notamment aux premiers pavillons romanis à la biennale de Venise (en 2007 et 2011), aux créations de l'International Romani Film commission, de l'European Roma Institute for Arts and Culture (ERIAC) et de la Roma Archive à Berlin, en 2017.

Partie prenante de ce mouvement de l'art contemporain romani bénéficiant d'une reconnaissance dans le courant des années $2000^{41}$, Delaine Le Bas et Damian James Le Bas élaborent une documentation filmique en regard de leurs œuvres consacrées à la mémoire de la circulation des Gypsies et Irish Travellers sur le territoire du RoyaumeUni. Cette mémoire familiale des routes, des chemins et lieux de rencontre des groupes romanis est au cœur de deux œuvres importantes élaborées sur près d'une décennie : To Gypsyland, une archive multimédia collectée et à l'occasion installée par Delaine Le Bas depuis le début des années 2000 (avec son mari Damian Le Bas, décédé en 2017), et The Stopping Places (2018), un ouvrage écrit sous la forme d'un carnet de route par Damian James Le Bas (son fils). En préparation de ces deux œuvres, les artistes ont réalisé une série de courts métrages - London Gypsyland (2013), Glasgow Gypsyland (2013), Peterborough Gypsyland (2013), Chuvihoni (2014) - conçue comme un patchwork d'archives personnelles et de déambulations filmées avec des caméras numériques amateurs. 
Fig. 10

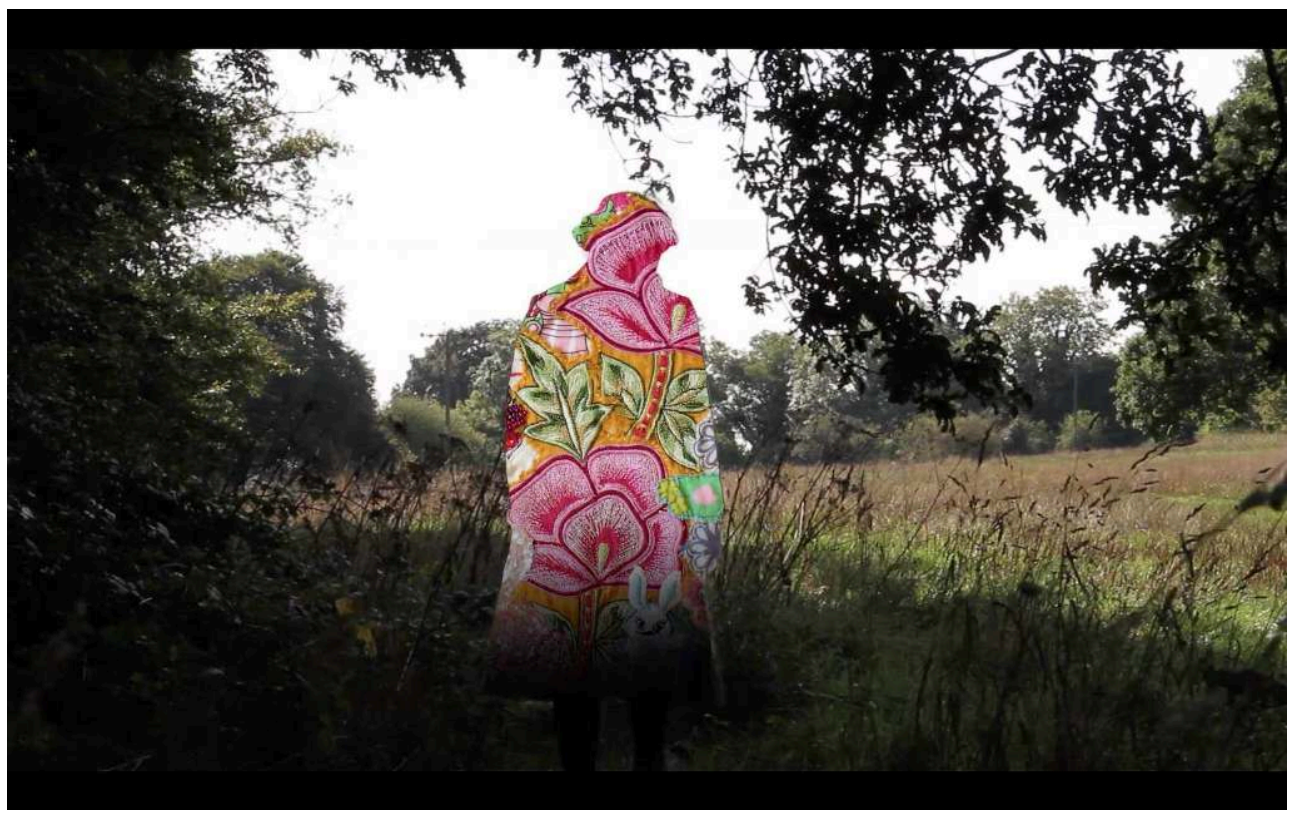

Delaine Le Bas et Damian James Le Bas, Chuvihoni, 2014

Fig. 11

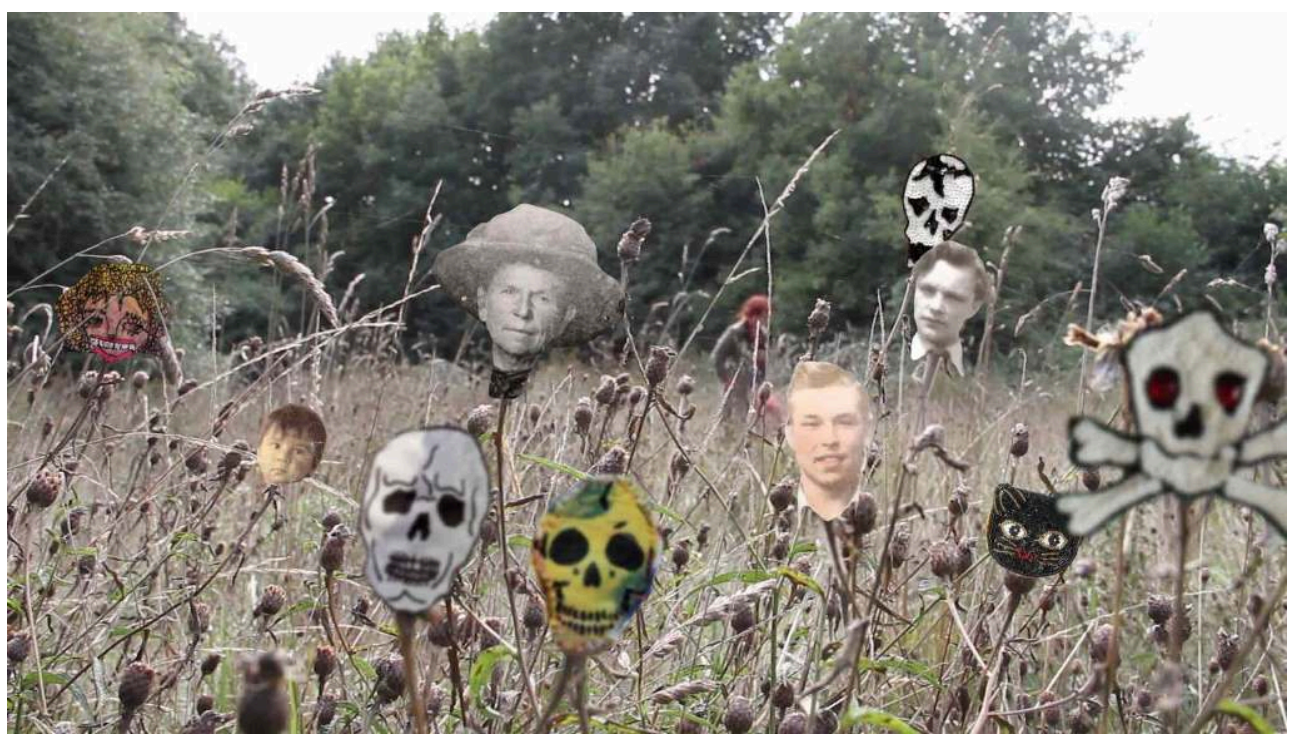

Delaine Le Bas et Damian James Le Bas, Chuvihoni, 2014

Dans leur court métrage Chuvihoni, Delaine et Damian James Le Bas subvertissent les inscriptions ésotériques, que l'«improbable ${ }^{42}$ écrivain et tsiganologue Jean-Paul Clébert prenait pour des survivances de l'ancien alphabet du romanès ${ }^{43}$. Passant outre la mésestime des tsiganologues et des intellectuels romanis pour les travaux de Clébert $^{44}$, les deux artistes réinscrivent ces fantasmagories dans une histoire visuelle personnelle, élaborée en regard de témoignages familiaux sur les "lieux de haltes » (stopping places - ou atchin tans en romanès) des Gypsies du sud de l'Angleterre. Par le remploi et le détournement, les deux artistes transforment ces prétendus vestiges de la sorcellerie et de l'écriture hiéroglyphique tsigane en simple ornement des histoires de 
fantômes partagées et vécues au sein de leur famille. Au-delà de ce travail de reprise iconographique, Chuvihoni est le lieu de reconstitution d'une histoire familiale. À partir d'un enregistrement sonore (difficilement audible) d'une conversation de Delaine avec Nan, sa grand-mère, le film présente les lieux où elle et les siens avaient pour habitude de s'installer pour un temps, avant qu'ils ne se sédentarisent il y a 70 ans. Alternant entre un espace domestique désert et celui d'une rase campagne du Sud de l'Angleterre, les plans (fixes pour la plupart) sont progressivement mis en mouvement par des éléments qui traversent l'image en surimpression: hiéroglyphes tirés de l'œuvre de Jean-Paul Clébert, portraits et photographies de famille découpés au ciseau, figurines réalisées en broderie ou à l'aquarelle et animées par un processus de compositing (réalisé par une collaboratrice) (Fig. 10 et 11).

Le tissage entre les images et les sons, avec l'écho lointain de bruitages sonores et la voix sourde de Nan, rend progressivement compte d'une mémoire des « lieux de halte » des Travellers, une mémoire dénuée des motifs des caravanes et du feu de camp - des archétypes absents du film si ce n'est la cheminée d'une cuisine intérieure en introduction du film. Les artistes figurent ainsi (en tout juste cinq minutes) des lieux habités par des expériences vécues de la famille, des noms, des rêves et des histoires. Incarné par toutes ces figures et figurines qui semblent être exhumées de terre et imprégner l'atmosphère, ce travail quasi archéologique esquisse de manière complémentaire les méandres de la mémoire familiale et de territoires singuliers. Si le film n'est introduit par aucun texte explicatif, Damian James Le Bas évoque dans son livre ces espaces du Hampshire où ses ancêtres vivaient en partie de la cueillette du houblon, des lieux peu connus en comparaison avec les paysages de carte postale du Kent voisin :

Avec son réseau de fermes isolées, de plantations fruitières, de hameaux et de villages, la plupart des gens pensent qu'il s'agit d'une partie indéfinie de la campagne, un morceau d'Angleterre rurale qui n'a rien de particulier. Mais pour les Gypsies du coin, dont ma famille, cette région a un nom. C'est un nom qui rappelle pourquoi cet endroit est spécial, et pourquoi il est spécial pour nous. Nous l'appelons le Pays du Houblon ${ }^{45}$. 
Fig. 12

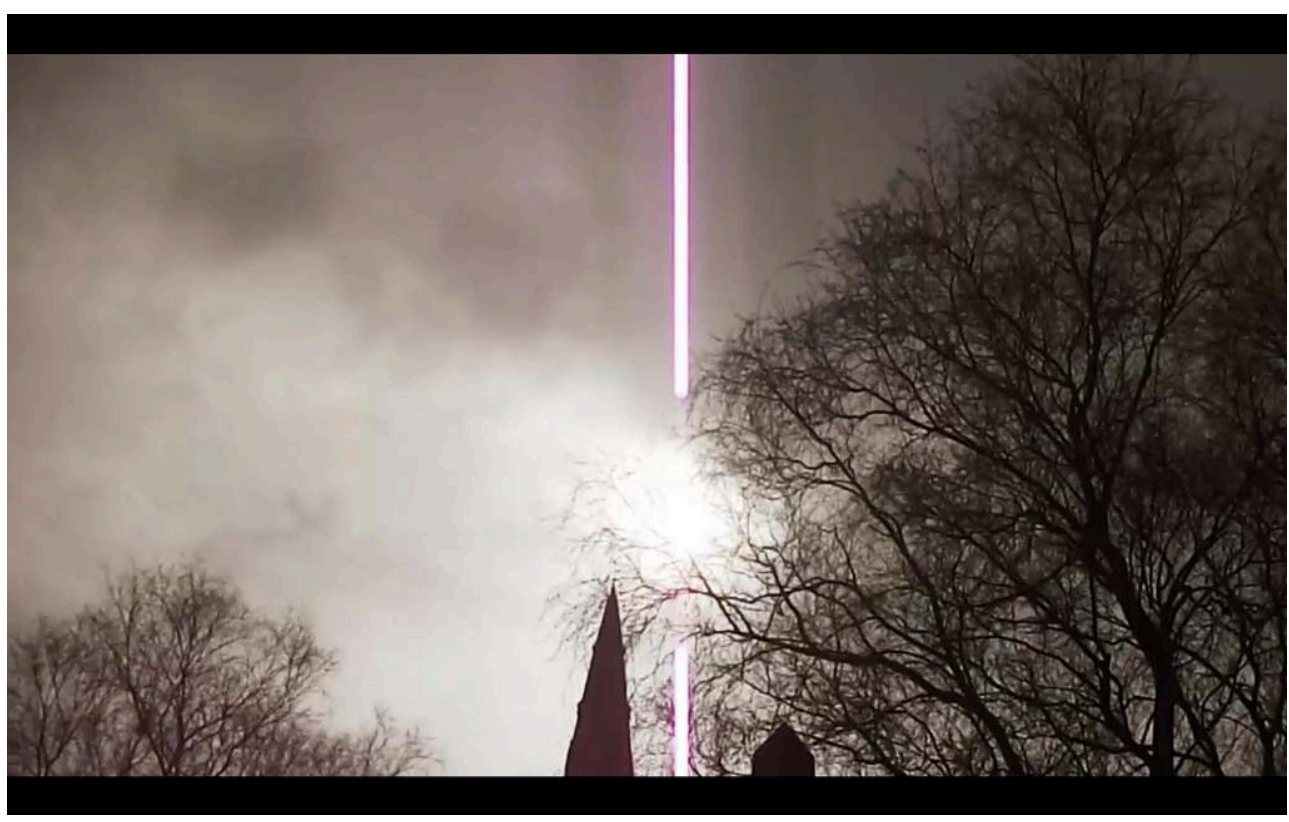

Delaine Le Bas, Glasgow Gypsyland, 2013.

Fig. 13

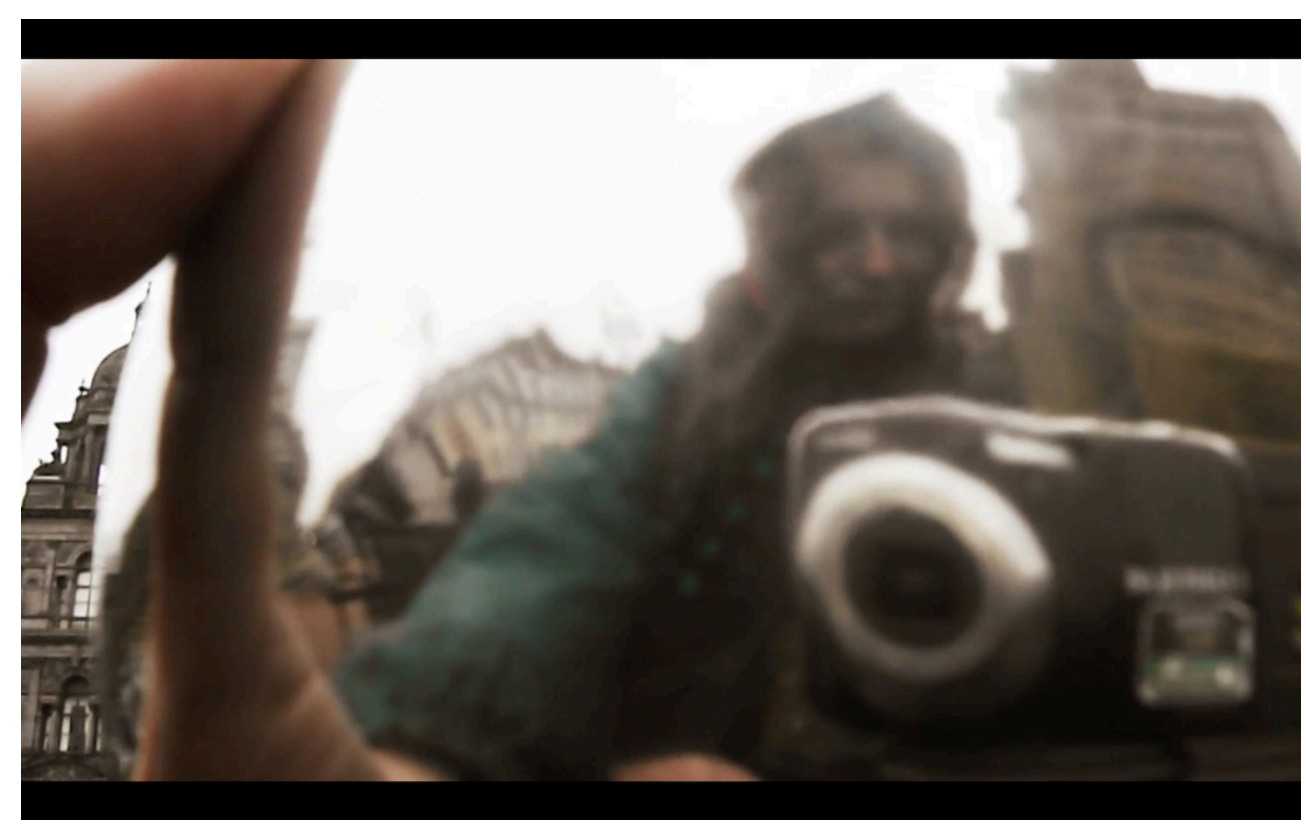

Delaine Le Bas, Glasgow Gypsyland, 2013

Chuvihoni s'inscrit dans un projet bien plus large de cartographie transmédiale des mondes romanis anglais qui conjugue un souci documentaire et les puissances de la fabulation (à l'image de cette association entre les hiéroglyphes imaginaires de clébert et les photographies de la famille Le Bas). Cette mise en commun d'une mémoire orale et d'un futurisme romani s'incarne tout particulièrement dans le projet To Gypsyland dédié à la mémoire et l'invention d'un monde gypsy et traveller, pour partie effacé, et dont il faut retrouver les traces, et pour partie imaginaire, mêlant art brut, dadaïsme et influence punk. Parmi les nombreux éléments de cette archive du Gypsyland, Delaine Le 
Bas a réalisé trois films : London Gypsyland (2013), Glasgow Gypsyland (2013), Peterborough Gypsyland (2013) avec un petit appareil photo numérique, de basse résolution, utilisé en mode vidéo. Les images muettes, avant d'être montées et sonorisées par Damien James Le Bas, sont réalisées selon la même procédure. Au fil de ses déambulations, l'artiste collecte l'ensemble des signes qui attestent du passage des familles romanis (Gypsies et Irish Travellers) tout en se mettant elle-même en scène avec le style et les costumes qui lui sont propres, faits de breloques, de couleurs, de foulards, se jouant des motifs que la société majoritaire associe aux Romanis. Faisant usage de toutes les « défectibilités » de l'image numérique produite par un appareil d'entrée de gamme (variation de la colorimétrie, pixels morts, fluctuation du signal vidéo [jitter], aberrations chromatiques), Delaine Le Bas associe ainsi son travail de collecte des traces relatives à la circulation des familles romanis dans les mondes ruraux et urbains d'Angleterre à une instrumentalisation de l'instabilité de l'image vidéo (Fig. 12 et 13). La mémoire - et la projection - des mondes romanis dans un cosmopolitisme européen passé et futur se complète ainsi toujours d'une mise à l'index du caractère éphémère et défaillant du médium filmique ${ }^{46}$.

\section{Conclusion}

Trop souvent réduit à une poignée de cinéastes reconnus, le cinéma romani est aussi, et peut-être même avant tout, un lieu d'expérimentation de formes en dehors des productions (et des conventions formelles et techniques) de l'industrie cinématographique ${ }^{47}$. Réalisé avec une parcimonie de moyens, dans une économie domestique faite de débrouille et de bricolages, ce cinéma romani a depuis les années 1960 accordé ses outils techniques et ses pratiques aux sujets filmés et aux thèmes traités : que ce soit par nécessité, comme c'est le cas de Matéo Maximoff, par souci d'établir une relation plus symétrique avec des personnes filmées vivant dans la précarité (Pilar Arcila), ou encore pour engager une réflexion sur l'obsolescence et la défectibilité des technologies filmiques de basse résolution (Ben Vine, Delaine Le Bas). L'étude de ces "formes vagabondes " dans le cinéma romani encourage à considérer avec davantage d'attention les accords (au sens de l'anglais attunement), les échos, les harmonies entre formes de vie, techniques filmiques et formats des images enregistrées.

L'observation des copies argentiques de Matéo Maximoff, la description de la genèse des films de Pilar Arcila, Delaine Le Bas, Damian James Le Bas, Ben Vine, inscrivent ce cinéma romani dans l'histoire des images pauvres et d'un "cinéma imparfait ", qui " réduit les distinctions entre auteur et audience, et combine la vie et l'art [et dont la] visualité - floue, amateur, pleine d'artefacts - est fortement compromise $»^{48}$. La réunion de ces films au sein d'un même corpus opère toutefois des déplacements par rapport à la généalogie tracée par Hito Steyerl. Alors que la théoricienne et cinéaste distingue très clairement les « images riches » du film argentique avec les "pauvres » répliques numériques, la présente étude s'est plutôt attachée à observer les pratiques partagées par les utilisateurs des deux médiums (film et vidéo). Des pratiques qui sont résolument attachées aux images de basse résolution: le Super 8, pour le film argentique, la MiniDV ou les images vidéo compressées, pour le numérique. Par son attention aux techniques, aux technologies et à la matérialité des supports d'enregistrement, ce travail s'inscrit ainsi dans le fil des format studies. À l'instar des travaux d'Alexandra 
Schneider sur les formats réduits utilisés pour la distribution domestique des films des industries culturelles ${ }^{49}$, les formes vagabondes du cinéma romani brouillent les cartes d'une histoire des techniques en cinéma conçue autour du contraste entre les formats et les médiums filmiques et vidéo, esquissant ainsi autant de chemins de traverse parmi nos catégories d'usage et d'analyse.

\section{NOTES}

1. Sur les enjeux d'une histoire des mondes romanis, distincte des simples points de vue de la société majoritaire, voir Ilsen ABOUT et Marc BORDIGONI, «Écrire une histoire des mondes tsiganes : la découverte et le soulèvement des archives ", dans Ilsen ABOUT et Marc BORDIGONI (dir.), Présences tsiganes. Enquêtes et expériences dans les archives, Paris, Le Cavalier bleu, 2018, p. 17-39

2. Une histoire engagée dans le domaine de la photographie par Ilsen About avec l'étude du "régime visuel d'exclusion » produit par la presse illustrée française au tournant du XIX et $\mathrm{Xx}^{\mathrm{e}}$ siècle: "Une fabrique visuelle de l'exclusion. Photographies des Tsiganes et figures du paria entre 1880 et 1914 », dans Catherine CoQuio et Jean-Luc PouEYTo (dir.), Roms, Tsiganes, Nomades. Un malentendu européen, Paris, Karthala, 2014, p. 431-446.

3. Hito STEYERL, «In Defense of the Poor Image », e-flux journal, 10, 2009, http://www.e-flux.com/ journal/in-defense-of-the-poor-image/ (consulté le 30/01/20).

4. Voir en particulier Alexandra Schneider et sa notion de "format de passage » à propos des copies réduites en petits formats (small-gauge reduction prints) utilisées pour la distribution des films en dehors des salles de cinéma: Alexandra SCHNEIDER, «Viewer's Digest: Small-Gauge and Reduction Prints as Liminal Compression Formats", dans Marek JANCovic, Axel Volmar et Alexandra SCHNEIDER (dir.), Format Matters: Standards, Practices, and Politics in Media Cultures, Lüneburg, Meson press, 2020, p. 129-146.

5. Thomas ELSAESSER, " Das Kino im 21. Jahrhundert - Kunstform oder Lebensform? ", Kracauer Lectures, 06 novembre 2012, https://doi.org/10.25969/mediarep/13718 (consulté le 15/05/2020) ; Marielle MACÉ, Styles. Critique de nos formes de vie, Paris, Gallimard, 2016.

6. L'ouvrage MP3 - the meaning of a format de Jonathan Sterne fait office d'ouvrage fondateur des format studies. La citation est extraite de la traduction française : MP3. Politiques de la compression, Philharmonie de Paris, 2018, p. 35. Sur l'émergence et l'actualité des études des formats dans le champ des études cinématographiques voir: M. JANCovic, A. VolmAR et A. SCHNEIDER (dir.), Format Matters, op. cit.

7. Voir par exemple Dina I ORDANOvA, «Welcome Pictures, Unwanted Bodies. "Gypsy" Representations in New Europe's Cinema ", dans Glajar VAlentinA et Domnica RAdULESCU (dir.), “Gypsies" in European Literature and Culture, New York, Pilgrave Macmillan, 2006, p. 235-240.

8. Ivan Illich, Le Travail fantôme, Paris, Seuil, 1981, p. 93 et 92.

9. Ethel BRooKs, "Reclaiming: The Camp and the Avant-Garde », dans Daniel B AKER et Maria HLAVAjova (dir.), We Roma : A Critical Reader in Contemporary Art, Utrecht, BAK, 2013, p. 114-138. La reconnaissance de l'art contemporain romani est marquée en 2007 par le premier pavillon rom (Lost Paradise) à la $52^{\text {ème }}$ Biennale de Venise.

10. Cette étude reprend les résultats d'une première recherche qui a abouti à la programmation du cycle «Romani cinema » à la Cinémathèque Française en 2016 et sur de nombreux échanges 
avec plusieurs artistes, notamment dans le cadre du séminaire « caméras politiques » co-organisé avec Perrine Poupin et Daniel Cefaï. Ce texte poursuit également une recherche en cours sur les archives filmiques inédites de Matéo Maximoff, confiée de manière temporaire à la Médiathèque Matéo Maximoff-Fnasat-Gens du Voyage. À cette fin, je veux vivement remercier Evelyne Pommerat, de m'avoir fait découvrir ce fonds audiovisuel sans égal, et Nouka Maximoff, pour m'autoriser à publier les photogrammes des films de son père.

11. Montage, réparation, projection des films, il maitrise toutes les techniques de la postproduction ; voir sur ce point la biographie atypique que lui consacre Gérard Gartner, biographie écrite à partir de ses souvenirs et du journal de Matéo Maximoff: Gérard GARTNER, Matéo Maximoff : carnets de route, Duravel, Alteredit, 2006.

12. Il bénéficie en janvier 1939 d'un non-lieu et est libéré immédiatement.

13. Une culture de contact qui s'inscrit dans une histoire remontant au XIX siècle et la proximité entre groupes romanis et bohème artistique, voir : Henriette Asséo, « Histoire des Bohémiens et Tsiganes en Europe ", dans Sylvain AMIc (dir.), Bohèmes. De Léonard de Vinci à Picasso, Paris, RMNGrand Palais, 2012, p. 26-35.

14. Émission du 25 octobre 1980. L'enregistrement sur bande magnétique est conservé dans le fonds audiovisuel, confié par Nouka Maximoff, sa fille, à la Médiathèque Matéo Maximoff-FnasatGens du Voyage.

15. C'est le terme qu'il utilise en 1983, alors qu'il se retrouve au micro de TSF 93, dans l'émission «Projecteur » en compagnie du sculpteur Gérard Gartner et du cinéaste Tony Gatlif (tous deux d'ascendance romani). Matéo Maximoff saluait alors la sortie du long-métrage Les Princes (1983), de Gatlif, comme le premier film réalisé par un cinéaste romani.

16. La mission est fondée en 1954 par le pasteur « gadjé » Clément Le Cossec.

17. Une pellicule positive, relativement peu coûteuse à l'époque.

18. Le dernier inventaire de Evelyne Pommerat, en janvier 2020, faisait une estimation de 7608 diapositives.

19. Je renvoie ici le lecteur aux analyses pionnières du photographe Antoine Le Roux, car nul doute que les motivations poussant Matéo Maximoff à apparaître à l'image sont multiples: «Force est de constater que toutes ces photographies trahissent l'œil particulièrement attentif de Maximoff d'une part à sa propre personne mais aussi à son environnement » (Antoine LE Roux, « Matéo Maximoff, écrivain et photographe ", Études tsiganes, n 60, 2017, p. 100).

20. Cela semble être le cas dans La bande à Matéo, un film sonore (dont le titre est incertain). Le montage des images présentant la vie quotidienne des membres de l'église de Noisy-le-Sec travail, loisir, moments de convivialité - est en effet ponctué par une prédication de Matéo Maximoff en voix-off.

21. "Vie et lumière » est le nom de l'association de la Mission évangélique des Tziganes de France qui semble avoir oublié l'existence de cette œuvre extraordinaire. Des jeunes responsables ont confirmé cette information à Evelyne Pommerat.

22. Sur « le processus continu de métamorphose médiatique de l'expérience pentecôtiste », en particulier dans sa dimension télévisuelle, je renvoie ici à l'excellente étude de Damien Mottier : «La main sur l'écran. Pentecôtisme et expérience du “toucher" télévisuel », Gradhiva, n²6, 2017, p. 33.

23. L'anthropologue Patrick Williams, qui a réalisé une monographie de cette Kumpania - Mariage tsigane. Une cérémonie de fiançailles chez les Rom de Paris, Paris, L'Harmattan, Selaf, 1984 - et connaissait très bien Matéo Maximoff, dresse un portrait sensible et juste de l'incroyable diversité de ses activités et de ses voyages dans un texte du volume de la revue Études Tsiganes qui lui a été consacré : « Le pasteur Matéo », Études tsiganes, n 60, 2017, p. 142-158.

24. Fragments du tapuscrit, p. 6. 
25. Que ce soit en photographie ou au cinéma, ils ont travaillé à la sauvegarde des pratiques argentiques : le site de Ben Vine est justement intitulé Analogue Resistance; Url : http:// www.analogueresistance.net/ (consulté le 25/06/21).

26. I. ILLICH, Le Travail fantôme, op. cit., p. 92.

27. Voir notamment le texte écrit par Ben Vine pour le cycle de programmation «Romani cinema » à la Cinémathèque ; Url : http://www.debordements.fr/Romani-Cinema-1-512 (consulté le $10 / 03 / 21)$.

28. J'entends ici par défectibilité («defect») les «signes et indications de limites des caractéristiques" [d'un support technique] et les "usages erronés d'un système d'enregistrement et d'une technologie ", Alessandro BoRDINA et Simone VeNTURINI, «Operational Practices for a Film and Video Preservation and Restoration Protocol », in Julia NooRDEGRAAF et al (dir.), Preserving and Exhibiting Media Art. Challenges and Perspectives, Amsterdam, Amsterdam University Press, 2013, p. 257.

29. Voir sur ce point la table ronde qui a réuni dans les pages d'un numéro d'October plusieurs acteurs de la programmation et de la création du cinéma expérimental aux États-Unis : CoLLECTIF, «Round Table. Obsolescence and American Avant-Garde Film », October, n 100, 2002, p. 115-132.

30. J'emprunte le terme "filmant " à Christian Lallier, dont les travaux et les films conçoivent «la situation sociale comme un lieu de travail où se construisent des logiques de pouvoir, des relations négociées et des formes de coopération à observer » (Christian LALLIER, Pour une anthropologie filmée des interactions sociales, Paris, Archives Contemporaines, 2009, p. 19).

31. Au moment des premiers tournages, les premières étapes de l'opération « Euroméditerranée 1 » avait commencé, avec notamment la démolition de nombreux immeubles dans les quartiers situés autour de la gare St Charles, de la Porte d'Aix et du Port Autonome.

32. Texte inédit de Pilar ARCILA et Jean-Marc LAMOURE, rédigé pour leur intervention au sein du séminaire « Caméras politiques », EHESS, séance du 22 mars 2017.

33. Idem.

34. Sur la genèse de cette représentation de la précarité comme consubstantielle aux groupes romanis, voir les nombreux travaux d'Ilsen About et notamment celui consacré à cette « fabrique visuelle de l'exclusion »; I. ABout, « Une fabrique visuelle de l'exclusion », op. cit.

35. Entretien avec Pilar Arcila, le 15 décembre 2015.

36. P. ARCILA et J.-M. LAMOURE, texte inédit, op. cit.

37. Jonathan L ARCHER et Nicole BRENEZ, «Romani Cinema. Figurative Defamations and Documented Rectifications ", Débordements, 24 juin 2015. Url: http://www.debordements.fr/ Romani-Cinema-1 (Consulté le 08/08/16).

38. Nicole BRENEZ, Manifestations. Écrits politiques sur le cinéma et autres arts filmiques, Réville, de l'incidence éditeur, 2019, p. 65.

39. Colin CLARK, " "If you build it..." On decolonising Romani knowledge, history and practice - the launch of the European Roma Institute for Arts and Culture ", Coalition for Racial Equality and Rights. Url: https://www.crer.scot/post/2017/06/08/guest-blog-ifyou-build-it-on-decolonising-romani-knowledge-history-and-practice-the (consulté le 15/06/17).

40. Anna Mirga-Kruszelnicka, "Romani Studies and emerging Romani scholarship ", Roma Rights, $\mathrm{n}^{\circ} 2$, 2015, http://www.errc.org/roma-rights-journal/roma-rights-2-2015nothing-about-us-without-us-roma-participation-in-policy-making-and-knowledgeproduction (consulté le 12/03/21).

41. Cette chronologie de l'art contemporain se concentre sur la formation de collaborations et d'institutions européennes. L'exposition des travaux des artistes visuels roms s'observe, à l'échelle nationale, dès les années 1970, notamment en Hongrie avec The First National Exhibition of 
Self-Taught Rom Artists organisée par l'Institut Hongrois de la Culture en 1979, voir à ce propos Ágnes DARóczI, «The Birth of Roma Visual Arts: Hungary, 1979 », in D. BAKER et M. HLAVAJOVA (dir.), We Roma, op. cit., p. 140-150.

42. Ian HANCOCK, "The Concoctors : Creating Fake Romani Culture", in Susan TEBBUTT et Nicholas SAUL (dir.), The Role of the Romanies: Images and Counter-images of 'Gypsies'/Romanies in European Culture, Liverpool, Liverpool University Press, 2004, p. 86.

43. Voir en particulier son ouvrage Les Tziganes, Paris, Arthaud, 1961.

44. Le travail de Jean-Paul Clébert relève, dans l'histoire de la connaissance des groupes romanis, des " grandes synthèses universelles » - spéculatrices ou fabulatrices - qui dominaient le champ jusqu'aux premières études historiques circonscrites et documentées émergeant au milieu des années 1970. Sur cette histoire intellectuelle, voir : I. ABOUT et M. BORDIGONI (dir.), «Écrire une histoire des mondes tsiganes ", op. cit., p. 17.

45. «With its sparse network of scattered farms, fruit plantations, and outlying hamlets and villages, it would seem to most people to be a nondescript part of the countryside, a bit of rural England that's not really special in any particular way. But for the local Gypsies, my family among them, this area does have a name. It's a name that reminds why this place is special, and why it is special to us. We call it Hop Country » (Damian LE BAS, The Stopping Places. A Journey through Gypsy Culture, Londres, Chatto \& Windus, 2018, p. 39). Traduction de l'auteur.

46. Lors d'un entretien avec Damian James et Phillip Osborne en juin 2015, Damian me racontait avec un sourire malicieux comment la basse résolution des images d'un film précédent de Delaine (Acimasko tan (Stopping places), 2012) avait provoqué la consternation de l'équipe technique d'un festival de cinéma qui l'avait programmé.

47. Jusqu'au début des années 2010, cette réduction s'observe même parmi les artistes romanis. Comme me l'a précisé Damian James Le Bas, «[...] en février 2012, lors de la création de la Commission International du Film Romani (International Romani Film Commission) [...] chacun pensait qu'il n'existait que deux cinéastes romanis : Tony Gatlif, et lui-même »; Entretien avec Damian James Le Bas et Phillip Osborne, le 11 juin 2015.

48. Julio García ESPINOSA, «For an imperfect cinema », Jump Cut, n² 20, 2005 [1979], p. 24-26, http://www.ejumpcut.org/archive/onlinessays/JC20folder/ImperfectCinema.html (consulté le 12/02/21). Cité par H. STEYERL, «In Defense of the Poor Image », op. cit.

49. A. SCHNEIDER, «Viewer's Digest: Small-Gauge and Reduction Prints as Liminal Compression Formats », op. cit.

\section{RÉSUMÉS}

Dans une perspective qui allie histoire du cinéma et anthropologie des Roms/Tsiganes, ce texte propose une analyse des pratiques et des contraintes techniques éprouvées par les artistes et les cinéastes, amateurs ou professionnels, pour décrire la mobilité et les conditions de vie des populations romanis en Europe de l'Ouest depuis 50 ans. Les films de cette mémoire, de cette vie d'itinérance, de migration et de circulation font ainsi correspondre ces formes de vie avec les techniques et formats filmiques utilisés. Cette généalogie d'un cinéma romani fait de bricolages, de vidéos de basse résolution et d'« images pauvres » (Steyerl) est une contribution directe à une histoire visuelle des mouvements politiques romanis et aux récents travaux qui, dans le champ des études cinématographiques, appréhendent les pratiques filmiques comme un écosystème. 
From a perspective that combines film history and the anthropology of Roma/Gypsies, this text proposes an analysis of the practices and technical constraints experienced by artists and filmmakers, both amateur and professional, in describing the mobility and living conditions of the Roma populations in Western Europe over the past 50 years. The films of this historical experience, of this life of itinerancy, of migration and circulation, necessarily match these forms of life with the particular utilitarian filmic techniques and formats. This genealogy of a Romani cinema - made of bricolage, low-resolution videos and "poor images" (Steyerl) - is a direct contribution to a visual history of Romani political movements and to recent works in the field of film studies that apprehend filmic practices as an ecosystem.

\section{INDEX}

Mots-clés : Film, technique, Roms/Tsiganes, média, histoire du cinéma, mouvement politique, migration, nomadisme

Keywords : Film, technique, Roma/Gypsies, media, film history, social movement, migration, nomadism

\section{AUTEUR}

\section{JONATHAN LARCHER}

Anthropologue et cinéaste, Jonathan Larcher est post-doctorant à l'Eur ArTeC (UPL). Il a obtenu son doctorat en anthropologie à l'EHESS (Paris) et a été post-doctorant au New Europe College (Bucarest). Après avoir mené une longue enquête ethnographique dans un quartier rom en Roumanie, son travail de recherche et de création explore les questions épistémologiques et méthodologiques soulevées en anthropologie par les pratiques audiovisuelles, les images vernaculaires et les archives. Il travaille actuellement sur un projet de recherche consacré à la numérisation et à la préservation de l'archive vidéo du collectif politique Promedios (San Cristobal de Las Casas, Mexique). 